\title{
Endoscopic Mucosal Resection versus Endoscopic Submucosal Dissection for Large Polyps: A Western Colonoscopist's View
}

\author{
Ian Holmes ${ }^{1}$ and Shai Friedland ${ }^{2}$ \\ ${ }^{1}$ Department of Gastroenterology, Stanford University School of Medicine, Stanford, CA, ${ }^{2}$ VA Palo Alto Health Care System, Palo Alto, CA, \\ USA
}

To discuss the rationale for the widespread application of endoscopic mucosal resection (EMR) rather than endoscopic submucosal dissection (ESD) in Western centers. In Western centers, EMR is the treatment of choice for most non-pedunculated colorectal adenomas $>2 \mathrm{~cm}$ in size. EMR is sufficiently effective and safe to be performed without post-procedure hospitalization. Advances in EMR have led to reduced recurrence rates, and recent studies have demonstrated excellent outcomes with endoscopic treatment of recurrent adenomas. While studies from Asia have demonstrated lower recurrence rates with ESD, concern about the higher perforation risk and lengthy procedure time of ESD are two of the barriers preventing widespread adoption of ESD in the West. EMR is likely to continue as the dominant method for the treatment of large colorectal adenomas in Western centers until the limitations of ESD are overcome. Clin Endosc 2016;49:454-456

Key Words: Colonic polyps; Colonoscopy; Colorectal neoplasms

\section{INTRODUCTION}

Endoscopic mucosal resection (EMR) is widely used in Western centers for the treatment of non-pedunculated colorectal adenomas $>2 \mathrm{~cm}$ in size. Colonic endoscopic submucosal dissection (ESD) for the treatment of these adenomas is rarely performed outside of Japan and Korea. ${ }^{1}$ EMR is associated with a low risk of adverse events, rapid procedure times, and relatively low technical complexity; it is also suitable for outpatient treatment as patients generally only need to be observed for a few hours after the procedure and can go home the same day. ${ }^{2}$ ESD is technically complex, involves a long procedure time, and is associated with a significant risk of intraprocedure and delayed colon perforation; patients re-

Received: May 27, 2016 Revised: July 3, 2016

Accepted: July 13, 2016

Correspondence: Shai Friedland

VA Palo Alto Health Care System, 711 Middlefield Road, Palo Alto, CA 94301, USA

Tel: +1-650-787-7099, Fax: +1-650-321-7917, E-mail: shai_friedland@yahoo.com

(c) This is an Open Access article distributed under the terms of the Creative Commons Attribution Non-Commercial License (http://creativecommons.org/ licenses/by-nc/3.0) which permits unrestricted non-commercial use, distribution, and reproduction in any medium, provided the original work is properly cited. quire hospitalization for observation after the procedure. ${ }^{3}$ The main advantage of ESD is the excellent rate of en bloc resection, which reduces local recurrence rates and ensures precise histopathologic staging if malignancy is detected. However, advances in endoscopic optics have improved the visual assessment of malignancy, and improvements in endoscopic techniques have significantly reduced the local recurrence rate after EMR and facilitated the treatment of recurrences. EMR is therefore likely to continue as the widely used technique for the treatment of large colorectal adenomas in the West.

\section{EMR OUTCOMES IN WESTERN CENTERS}

\section{Outcomes of EMR}

Multiple large observational studies have documented the safety and efficacy of EMR for the treatment of non-pedunculated colorectal adenomas $>2 \mathrm{~cm}$ in size. The prospective multicenter Australian Colonic EMR (ACE) study demonstrated an intention-to-treat rate of $84 \%$ of successful EMRs with a mean procedure duration of 25 minutes. ${ }^{2}$ Submucosal invasive cancer was found in $6.9 \%$ of the cases and was generally treated with surgery. The adverse event rate was 
7.7\%: $2.1 \%$ of the cases required overnight hospitalization for post-procedure pain, $1.5 \%$ required a 3 to 5 day hospitalization and antibiotics for serositis, $2.9 \%$ required hospitalization for post-procedure bleeding, and $1.3 \%$ developed a perforation. A subsequent publication that included more patients and more extensive follow-up demonstrated a local recurrence rate of $16 \%$ at 4 -month follow-up, with an additional $4 \%$ recurrence rate found at 16 months. ${ }^{4}$

Risk factors associated with recurrence include lesion size $>40 \mathrm{~mm}$; use of argon plasma to ablate residual adenoma not amenable to snare resection; and prior manipulations leading to fibrosis, such as partial snare polypectomy, tattoo application at the site of the lesion, or aggressive biopsies of the lesion. $^{3-5}$

\section{New Advances in EMR}

Increased experience and improved endoscopic imaging using higher resolution cameras, dual focus design allowing closer inspection, and proprietary imaging techniques (narrow band imaging, blue laser imaging, and iScan) show significant promise in improving the endoscopic differentiation of benign and malignant colorectal lesions. ${ }^{6,7}$ This may influence treatment planning, as piecemeal resection with EMR is generally considered acceptable for benign adenomas, but en bloc resection should be performed for malignancies.

Avulsion, a newly described technique utilizing hot biopsy forceps with cutting current, enables complete removal of many fibrotic islands of residual adenoma encountered during EMR. In a comparative analysis involving argon plasma coagulation, the use of avulsion resulted in significantly higher rates of complete removal of all visible neoplasia during EMR and markedly reduced recurrence rates. ${ }^{8}$

Thermal ablation of the margin of the resection site after EMR can potentially reduce the incidence of recurrence by ablating microscopic areas of non-visible residual adenoma. Preliminary results from a multicenter randomized study presented in abstract form in 2016 demonstrated a reduction in adenoma recurrence from $20 \%$ to $6 \%$ with thermal ablation of the margin. ${ }^{9}$

Underwater EMR is another recently described technique in which colorectal adenomas are removed using a snare underwater, without submucosal injection. ${ }^{10}$ Underwater EMR is particularly well suited for non-lifting lesions with fibrosis from prior incomplete resection attempts or other manipulations. In this setting, it increases the complete resection rate of visible neoplasia and reduces recurrence. ${ }^{11}$ Successful underwater en bloc snare resection of 2- to 4-cm large adenomas has also been reported, challenging the conventional approach of avoiding en bloc snare resection of lesions $>2 \mathrm{~cm}$ due to the fear of perforation. ${ }^{12}$ If ongoing studies confirm the excellent efficacy and safety profile of this initial report, underwater en bloc resection may become more widely adopted.

\section{TREATMENT OF RECURRENCES AFTER EMR}

The majority of recurrences after EMR are unifocal and $<5$ $\mathrm{mm}$ in size. ${ }^{4}$ Over $90 \%$ of the recurrences can be treated with conventional snare polypectomy, EMR, or ablation. ${ }^{4}$ A second recurrence is found in $10 \%$ to $40 \%$ of all patients after treatment of the first recurrence. ${ }^{4,11}$ Underwater EMR appears to be superior to conventional EMR for treating recurrences, with a higher rate of complete removal of visible neoplasia and a lower second recurrence rate. ${ }^{11}$ Even lesions that recur two or more times can be treated successfully with additional EMR or underwater EMR. ${ }^{13}$ Treatment of recurrences with EMR or underwater EMR appears to be as safe as the treatment of naive lesions, with a low risk of bleeding or perforation. ${ }^{4,11,13}$ Progression to malignancy during treatment is very rare. ${ }^{4,13}$

\section{COMPARISON OF EMR AND ESD}

In expert centers, ESD enables complete en bloc resection of $>85 \%$ of all colorectal adenomas and superficial cancers. ${ }^{3}$ In contrast, en bloc resection rates for colorectal adenomas $>2$ $\mathrm{cm}$ in size are typically $25 \%$ or less with EMR. ${ }^{8}$ Adenoma recurrence is significantly more common with EMR than with ESD: recurrence rates for adenomas $>2 \mathrm{~cm}$ in size treated with EMR are typically approximately $16 \%$ in Western centers ${ }^{4}$ and $7 \%$ in Japanese centers, ${ }^{13}$ while recurrence rates with ESD are $1.4 \%$ in Japan. ${ }^{13}$ However, ESD requires significantly longer time to perform than EMR: the mean procedure time for EMR in a large Western study was 25 minutes while that for ESD in a large Japanese study was 116 minutes. ${ }^{2,3}$ In the same two studies, perforation occurred in $1.3 \%$ of the patients undergoing EMR and in $4.9 \%$ of the patients undergoing ESD. Hospitalization was required in $<10 \%$ of the Western patients who underwent EMR, while $100 \%$ of Japanese patients undergoing colorectal ESD are typically hospitalized for $>3$ days. ${ }^{14}$

While piecemeal EMR of adenomas results in good clinical outcomes in most patients, en bloc resection with ESD is preferable for cancers with slight submucosal invasion and favorable histologic features, as it provides curative endoscopic treatment without the need for surgery. ${ }^{15}$ Piecemeal resection with EMR may reduce the accuracy of histologic analysis and may lead to poor oncologic outcomes due to local recurrence. With current technology, endoscopic differentiation between adenoma and cancer is only $70 \%$ to $90 \%$ accurate, and the 
accuracy of differentiating between slight and deep submucosal invasion is only $70 \%$ to $80 \%$; therefore, precise histologic staging with an en bloc resection using ESD is preferable for lesions that have a significant likelihood of being invasive cancers. $^{15}$

\section{CONCLUSIONS}

EMR is safe and effective for treating colorectal adenomas. Compared with ESD, EMR is significantly faster to perform, has a much lower perforation rate, and does not require post-procedure hospitalization. Advances in EMR techniques have led to reduced local recurrence rates, and the modern treatment of recurrences is technically simple, safe, and usually effective. While ESD in expert centers still offers a superior technical result and lower recurrence rate, the procedural complexity, additional unreimbursed procedure time, perforation risk, and requirement of post-procedure hospitalization make it unlikely to be widely adopted in the West for treatment of colorectal adenomas. Improvements in endoscopic visual assessment of early submucosal invasive cancer may enable identification of a limited number of patients who may be suitable candidates for ESD in Western centers, if additional data showing safety and long-term efficacy comparable to surgery become available.

Conflicts of Interest

The authors have no financial conflicts of interest.

\section{REFERENCES}

1. Klein A, Bourke MJ. Advanced polypectomy and resection techniques. Gastrointest Endosc Clin N Am 2015;25:303-333.

2. Moss A, Bourke MJ, Williams SJ, et al. Endoscopic mucosal resection outcomes and prediction of submucosal cancer from advanced colonic mucosal neoplasia. Gastroenterology 2011;140:1909-1918.
3. Saito Y, Uraoka T, Yamaguchi Y, et al. A prospective, multicenter study of 1111 colorectal endoscopic submucosal dissections (with video). Gastrointest Endosc 2010;72:1217-1225.

4. Moss A, Williams SJ, Hourigan LF, et al. Long-term adenoma recurrence following wide-field endoscopic mucosal resection (WF-EMR) for advanced colonic mucosal neoplasia is infrequent: results and risk factors in 1000 cases from the Australian Colonic EMR (ACE) study. Gut 2015;64:57-65.

5. Kim HG, Thosani N, Banerjee S, Chen A, Friedland S. Effect of prior biopsy sampling, tattoo placement, and snare sampling on endoscopic resection of large nonpedunculated colorectal lesions. Gastrointest Endosc 2015;81:204-213

6. Burgess NG, Pellise M, Nanda KS, et al. Clinical and endoscopic predictors of cytological dysplasia or cancer in a prospective multicentre study of large sessile serrated adenomas/polyps. Gut 2016;65:437-446.

7. Rey JW, Kiesslich R, Hoffman A. New aspects of modern endoscopy. World J Gastrointest Endosc 2014;6:334-344.

8. Holmes I, Kim HG, Yang DH, Friedland S. Avulsion is superior to argon plasma coagulation for treatment of visible residual neoplasia during EMR of colorectal polyps (with videos). Gastrointest Endosc 2016 Apr 11 [Epub]. http://dx.doi.org/10.1016/j.gie.2016.03.1512.

9. Klein A, Jayasekeran V, Hourigan LF, et al. A multi-center randomized control trial of thermal aablation of the margin of the post endoscopic mucosal resection (EMR) mucosal defect in the prevention of adenoma recurrence following EMR: preliminary results from the "SCAR" study. Gastroenterology 2016;150(4 Suppl 1):S1266-S1267.

10. Binmoeller KF, Weilert F, Shah J, Bhat Y, Kane S. "Underwater" EMR without submucosal injection for large sessile colorectal polyps (with video). Gastrointest Endosc 2012;75:1086-1091.

11. Kim HG, Thosani N, Banerjee S, Chen A, Friedland S. Underwater endoscopic mucosal resection for recurrences after previous piecemeal resection of colorectal polyps (with video). Gastrointest Endosc 2014;80:1094-1102.

12. Binmoeller KF, Hamerski CM, Shah JN, Bhat YM, Kane SD, Garcia-Kennedy R. Attempted underwater en bloc resection for large (2-4 $\mathrm{cm}$ ) colorectal laterally spreading tumors (with video). Gastrointest Endosc 2015;81:713-718.

13. Oka S, Tanaka S, Saito Y, et al. Local recurrence after endoscopic resection for large colorectal neoplasia: a multicenter prospective study in Japan. Am J Gastroenterol 2015;110:697-707.

14. Tomiki Y, Kawai M, Takehara K, et al. Clinical pathway to discharge 3 days after colorectal endoscopic submucosal dissection. Dig Endosc 2015;27:679-686.

15. Tanaka S, Kashida H, Saito Y, et al. JGES guidelines for colorectal endoscopic submucosal dissection/endoscopic mucosal resection. Dig Endosc 2015;27:417-434. 